\title{
Étude théorique de l'équilibre élastoroptrastique d'un sol pulvérulent autour du pressiomètre
}

\author{
J. MONNET \\ J. KHLIF \\ IRIGM \\ Université \\ Joseph-Fournier \\ B.P. 53 X \\ 38041 Grenoble
}

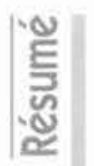

Cet article analyse le comportement tridimensionnel du sol pulvérulent autour du pressiomètre. Il est divisé en trois parties :

1) Dans la première partie, nous décrivons la loi de comportement du sol. Le sol est supposé suivre un modèle élasto-plastique non standard. Il est non cohérent et se dilate en plasticité, ce qui correspond à un comportement drainé. Nous prenons en compte la contrainte verticale. Nous montrons qu'il peut exister deux zones plastiques différentes autour du pressiomètre. La première zone est liée à une plasticité de cisaillement entre les contraintes circonférentielles et radiales. La seconde zone est liée à une plasticité de cisaillement entre les contraintes verticales et circonférentielles. Un équilibre élastique se développe au-delà des zones plastiques. Nous mettons en évidence la valeur des deux rayons plastiques, la relation entre les contraintes, déformations et déplacements dans chaque zone.

2) Dans la seconde partie, nous analysons des essais pressiométriques Ménard pour en tirer les caractéristiques mécaniques du sol. Nous comparons les résultats analytiques et expérimentaux.

3) Dans la troisième partie, nous calculons avec un programme aux éléments finis, l'équilibre autour du pressiomètre. Le programme utilise la loi de comportement MCK (Monnet, 1992). La comparaison entre les résultats analytique et numérique est faite, et nous concluons sur la validité de la méthode d'estimation des paramètres mécaniques.

\section{Theoretical study of the static equilibrium of the frictional soil around pressuremeter}

This paper analyses the equilibrium around the pressuremeter It is divided in three parts

1) In the first part, we describe the soil behaviour law. The soll is assumed to be elasto-plastic with non-associated plastic flow

The soil is non cohesive, and shows dilatancy in plasticity. This is a drained behaviour. We take into account the vertical stress. We show that two different plastic zones may developped around the pressuremeter. The first one is linked to the circumferential stress and the radial stress. The second one is linked to the vertical stress and to the circumferential stress. The elastic equilibrium beyond the plastic area is used for the analysis. We derive the expression of the two plastic radii, the relation between stress and strain in each zone and the expression of displacements.

2) In the second part, we use transform Ménard tests to determine the mechanical characteristic of the soil. We compare the analytical and experimental results.

3) In the third part, we compute with a finite element program the equilibrium around the pressuremeter. The program uses the behaviour law MCK (Monnet, 1992). The comparison between theoretical and experimental results for the stress distribution is shown, and we conclude on the method used to find the mechanical parameters.
} 


\section{Introduction}

Le pressiomètre inventé par Louis Ménard (1955 et 1959) est un appareil massivement utilisé de nos jours dans les projets de fondations. Son utilisation s'est largement étendue grâce aux travaux de Michel Gambin (1963 et 1979). Elle consiste habituellement à tirer de l'essai d'une part le module pressiométrique, et d'autre part la pression limite. Ces paramètres sont ensuite rentrés dans des tableaux de corrélation pour déterminer la contrainte admissible et le tassement des fondations. tions :

Cette méthodologie souffre de plusieurs imperfec-

- le module pressiométrique n'est pas une caractéristique mécanique intrinsèque au sol, mais est liée au terrain et à l'appareillage utilisé, ainsi qu'au mode de réalisation du forage :

- il y a une certaine imprécision dans l'estimation de la contrainte admissible et du tassement des fondations, lorsqu'on utilise des corrélations, ce qui peut conduire à des surcoûts de dimensionnement.

Cependant, le pressiomètre est le seul appareil d'essai in situ qui mesure à la fois une caractéristique de déformabilité et une caractéristique de résistance. Il permet de réaliser des mesures en place sur des sols non prélevables ou de forte granulométrie.

Cet article propose une interprétation complète des essais pressiométriques ou dilatométriques, ce qui permet dans le cas des sols pulvérulents :

- de déterminer leurs caractéristiques d'élasticité, et sous l'état de contrainte moyen rencontré ;

- de déterminer leur angle de frottement interne.

Ces deux caractéristiques sont propres au sol, indépendantes de la sonde ou du type de forage réalisé. Elles peuvent alors être prises en compte dans le projet sans passer par des corrélations toujours délicates. Le pressiomètre peut donc être utilisé pour d'autres travaux que ceux de fondation, et une interprétation soignée peut conduire à des économies considérables sur le dimensionnement.

Cette nouvelle méthodologie repose sur une procédure expérimentale plus complète, qui inclut dans la séquence de chargement un cycle de déchargerecharge, avant d'atteindre la pression de fluage. Ce cycle sert à déterminer les caractéristiques élastiques et permet d'éliminer la majeure partie des déformations plastiques. Le protocole expérimental comprend également un resserrement des mesures au-delà du fluage, de façon à obtenir plus de points expérimentaux dans cette zone de la courbe particulièrement fructueuse pour l'analyse. Il suppose une interprétation soignée des mesures de façon à prendre en compte tous les phénomènes hydrauliques et mécaniques qui se produisent pendant l'essai, tels qu'ils sont décrits dans le brevet Gaiatech (1989), et qui peuvent altérer la valeur de la pression de réaction du sol et la valeur de la déformation du rayon moyen de la sonde. Enfin, ce procédé utilise une analyse complète du comportement élasto-plastique du sol quand il est cisaillé par la sonde pressiométrique. Ce dernier aspect est présenté ici.
La démarche théorique consiste à prendre en compte une loi de comportement élasto-plastique linéaire pour le sol indiquée sur les figures 1 et 2, comme dans l'article de Hughes et al. (1977) sur l'essai pressiométrique dans le sable. Cependant, cette dernière étude souffre de deux simplifications. Tout d'abord une hypothèse de déformation plane est prise dans la direction verticale, et aucun déplacement n'est autorisé le long de cet axe. Par ailleurs, l'équilibre est seulement étudié dans le plan horizontal, et les forces verticales ne sont pas prises en compte. La possibilité d'une rupture se produisant entre les contraintes circonférentielles et verticales, de façon à ce que la contrainte radiale devienne intermédiaire, avait été envisagée par Wood et al. (1977), mais aucune formulation n'avait été proposée. L'étude présente est une tentative pour répondre à ces questions non résolues. Elle englobe le cas où la contrainte verticale constitue une direction de déformation plastique, et celui où elle ne joue aucun rôle.

La présente étude suppose pendant la phase élastique, qu'une contractance peut se produire. La phase plastique se développe avec une variation de volume. Le critère de plasticité dépend de l'angle de frottement interne $\Phi$, et l'écoulement plastique est lié au frottement intergranulaire $\Phi_{u}$. Ce modèle de comportement simple permet de représenter les sables et les sols pulvérulents en général. Le comportement drainé est pris en compte avec un coefficient de Poisson. L'analyse de l'équilibre se fait ici dans le plan horizontal, comme classiquement, mais aussi dans le plan vertical, de façon à tenir compte de l'influence éventuelle de la contrainte verticale. Suivant que la contrainte verticale intervient ou non dans la plasticité, on montre qu'il existe des formules différentes pour la pression de fluage (50) et (53), pour la pression limite (51) et (54), et pour l'équation de la courbe pressiométrique après le fluage (39) et (46). La méthode d'interprétation en cisaillement, consiste à reporter le logarithme népérien de la déformée du forage en fonction du logarithme népérien de la pression corrigée de réaction du sol. Les formules (38) et (45) montrent alors que cette relation est linéaire, avec une pente liée à l'angle de frottement interne et à l'angle de frottement intergranulaire, pour tous les points au-delà de la pression de fluage. Cette première détermination est affinée par la superposition sur le même graphique donné par l'ordinateur des courbes pressiométriques expérimentales et théoriques (39) et (46) en terme de pression en fonction de la déformée du forage. L'ajustement des deux représentations permet de déterminer définitivement l'angle de frottement interne du sol. La valeur des modules d'élasticité est déterminée à partir des cycles de déchargement-rechargement. L'expression des pressions limites conventionnelles est également fournie.

La vérification de cette nouvelle théorie se fait sur les essais pressiométriques réalisés lors de la campagne de reconnaissance complémentaire pour la construction du collecteur de Cauderan-Naujac à Bordeaux, dans les sables marins, et pour la construction du métro de Lyon sous la place Bellecour, dans les graves alluviales. Ces essais sont interprétés par le logiciel Gaiapres de façon à obtenir les pressions corrigées et les déformations moyennes du forage. On vérifie alors le bon accord des pressions limites théorique et expérimentale pour une expansion conventionnelle de la sonde. 


\section{NOTATIONS}

$\sigma_{1}, \sigma_{2}, \sigma_{3}$ contraintes principales

$\varepsilon_{1}, \varepsilon_{2}, \varepsilon_{3}$ déformations principales

$v$ coefficient de Poisson

E module de Young

$\lambda$ et $\mu$ coefficients de Lamé

$\Phi \quad$ angle de frottement interne

$\Phi_{\mu} \quad$ angle de frottement intergranulaire

$\Psi \quad$ angle de dilatance

$\sigma_{1} \quad$ contrainte de compression majeure

$\sigma_{3} \quad$ contrainte de compression mineure

$\sigma_{\theta} \quad$ contrainte circonférentielle

$\sigma_{\text {r }} \quad$ contrainte radiale

$\sigma_{\gamma} \quad$ contrainte verticale

$\varepsilon$ déformation radiale

$\varepsilon_{0} \quad$ déformation circonférentielle

$\varepsilon$. déformation verticale

$\gamma \quad$ poids volumique

a rayon du forage

b rayon externe de la première zone plastique

c rayon extene de la seconde zone plastique

$\sigma_{\text {c }} \quad$ contrainte radiale à la limite du domaine élastique (fin de la seconde zone plastique)

$\sigma_{\theta c} \quad$ contrainte circonférentielle à la limite du domaine élastique (fin de la seconde zone plastique)

$\mathrm{u}_{\mathrm{a}} \quad$ déplacement pour le rayon a du forage au niveau du pressiomètre

$\mathrm{u}_{\mathrm{b}} \quad$ déplacement pour le rayon $\mathrm{b}$

$u_{c} \quad$ déplacement pour le rayon $\mathrm{c}$

a. $\quad$ rapport $(1+n) /(1-N)$

\section{Étude analytique}

\section{Hypothèses}

Les contraintes sont négatives en compression et positives en traction,

Le sol est considéré avoir un comportement élastoplastique non standard représenté sur la figure 1. La partie élastique est liée aux constantes d'élasticité E (module de Young) et $v$ (coefficient de Poisson), qui permettent de définir les coefficients de Lamé $\lambda$ et $\mu$ :

$$
\lambda=E \cdot v /[(1+v) \cdot(1-2 \cdot v)] \quad \mu=E /[2 \cdot(1+v)]
$$

La partie plastique du comportement est liée aux deux angles de frottement $\Phi$ (angle de frottement interne), et $\Phi$ (angle de frottement intergranulaire ) dans le cas de l'essai triaxial de révolution, l'angle de frottement intergranulaire $\Phi_{u}$ est égal à l'angle $F_{f}$ de la théorie de Rowe (1969). L'écoulement plastique nonstandard utilise le scalaire $\xi$ qui définit la longueur de la déformation plastique non standard:

$$
\mathrm{d} \varepsilon^{p}=\xi \cdot \mathrm{d} G(\sigma) / \mathrm{d} \sigma
$$

avec la fonction non associée (4) où $\Psi$ est l'angle de dilatance du sol déterminé grâce à la relation de Monnet et al. (1978) comme la différence entre l'angle de frottement interne et l'angle de frottement intergranulaire :

$$
\begin{gathered}
\Psi=\Phi-\Phi_{\mu} \\
G(\sigma)=\left(\sigma_{3}-\sigma_{1}\right)+\sin \Psi \cdot\left(\sigma_{3}+\sigma_{1}\right)
\end{gathered}
$$
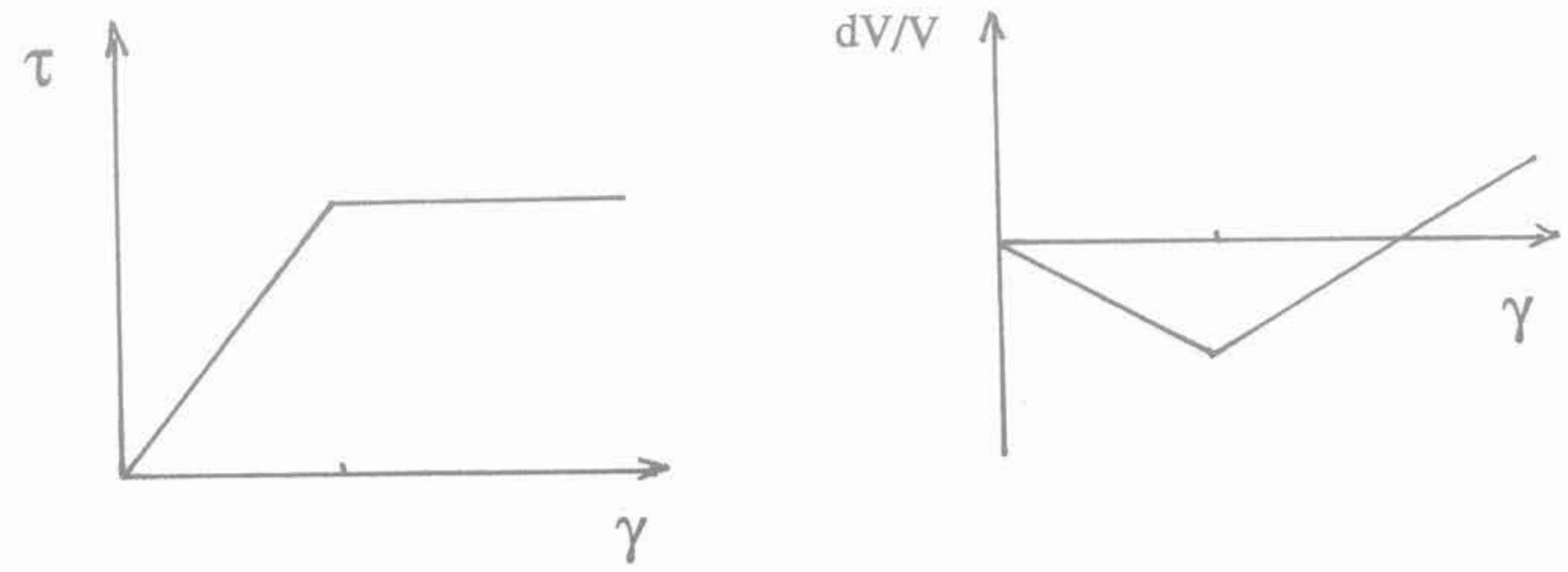

FIG.1 Comportement théorique du sol au cisaillement. Theoretical shearing behaviour of the soil.

Variation de volume théorique du sol au cisaillement.

Theoretical volume charge of soil when shearing occurs. 


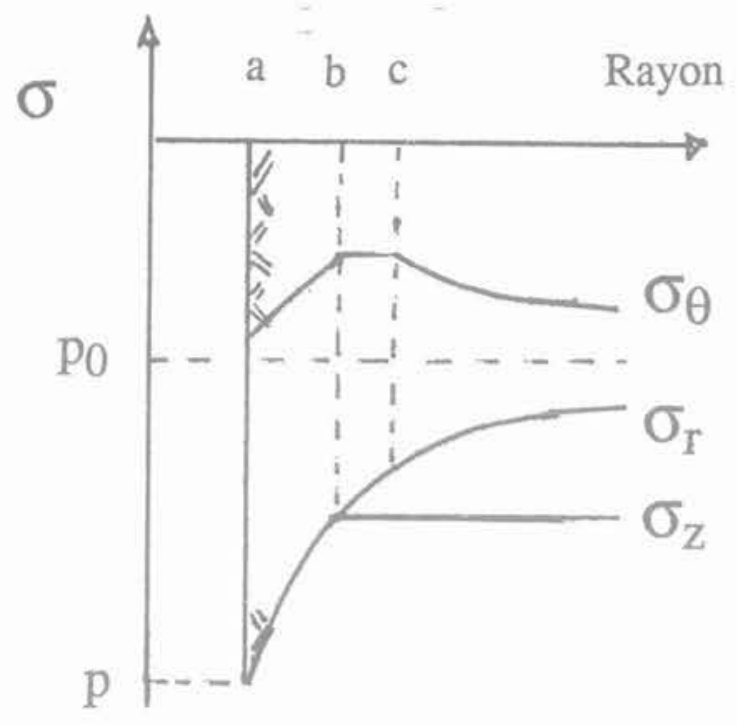

FIG.3 Premier cas : état des contraintes le long du rayon avec deux zones plastiques. First case : state of the stresses along the radius with two plastic zones.

L'équilibre-limite est atteint quand le critère de Mohr-Coulomb (5) s'annule:

$$
F(\sigma)=\left(\sigma_{3}-\sigma_{1}\right)+\sin \Phi \cdot\left(\sigma_{3}+\sigma_{1}\right)
$$

Trois différentes zones de sol sont considérées. Si nous partons de l'axe du forage (Fig. 3), nous trouvons : - une zone plastique dans laquelle la plasticité se produit entre la contrainte circonférentielle $\sigma_{\theta}$ et la contrainte radiale $\sigma_{r} c^{\prime}$ est-à-dire entre les rayons a (rayon du forage) et b (rayon externe de la première zone plastique) :

- selon les conditions de contraintes que nous expliciterons, une seconde zone plastique peut se développer entre les contraintes circonférentielles $\sigma_{0}$ et verticales $\sigma_{z}$ c'est-à-dire entre les rayons b (rayon externe de la première zone plastique) et c (rayon externe de la seconde zone plastique) (Fig. 3) ou bien cette zone n'existe pas (Fig. 4) :

- une zone élastique se développe au-delà.

\section{2}

\section{Conditions d'équilibre}

Dans le plan horizontal, la condition d'équilibre est:

$$
\sigma_{\theta}-\sigma_{f}-r \cdot \mathrm{d} \sigma_{\mathrm{r}} / \mathrm{dr}=0
$$

Dans le plan vertical, la condition d'équilibre est:

$$
\mathrm{do} / \mathrm{dz}=-\gamma
$$

\section{3}

\section{Première zone plastique}

Cette zone plastique est limitée par le rayon a du forage et du pressiomètre, et par le rayon b limite externe de la zone considérée. L'équilibre-limite se produit entre les contraintes circonférentielles $\sigma_{\theta}$ et radiales $\sigma_{\text {s }}$ selon le critère de Mohr-Coulomb (5), ce qui permet d'écrire :

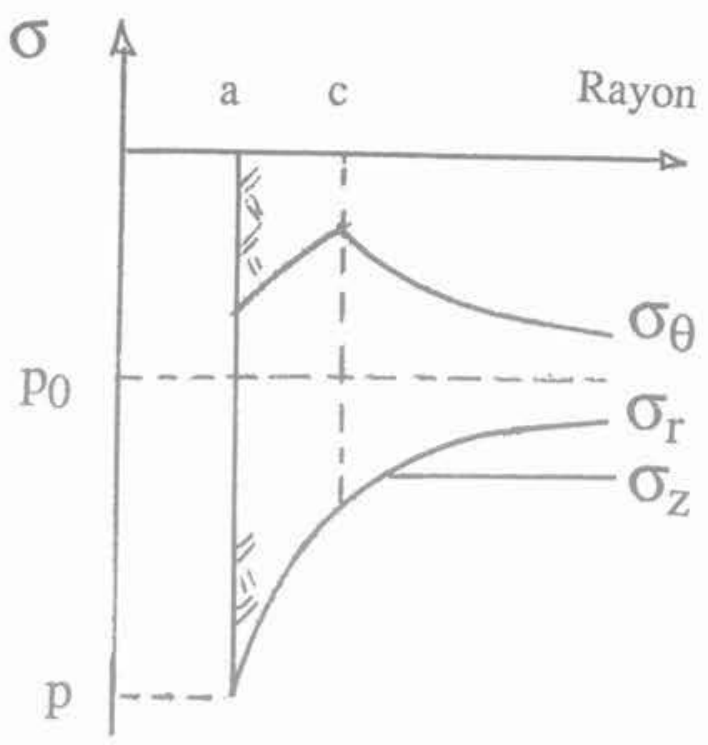

FG. 4 Second cas : état des contraintes le long du rayon avec une zone plastique. Second case : state of the stresses along the radius with one plastic zone.

avec :

$$
\sigma_{\theta} / \sigma_{\mathrm{T}}=\mathrm{N}
$$

$$
\mathrm{N}=(1-\sin \Phi) /(1+\sin \Phi)
$$

La condition d'équilibre (6) peut être écrite en fonction du rapport $\mathrm{N}$ des contraintes et intégrée entre les valeurs a du rayon du forage où la pression p est appliquée, et le rayon $r$ quelconque, interne à la première zone plastique où la contrainte radiale $\sigma_{t}$ s'applique. On en déduit la relation (10) :

$$
\operatorname{Ln}(\mathrm{r} / \mathrm{a})=1 /(\mathrm{N}-1) \cdot \operatorname{Ln}\left(\sigma_{\mathrm{r}} / \mathrm{p}\right)
$$

La condition de plasticité non standard (2), (3) et (4) montre que le rapport entre les incréments de déformation plastique radiale $\varepsilon_{f}$ et circonférentielle $\varepsilon_{\theta}$ est constant :

$$
\mathrm{d} \varepsilon_{r}{ }^{P} / \mathrm{d} \varepsilon_{\theta}{ }^{\mathrm{P}}=-\mathrm{n}
$$

avec:

$$
\mathrm{n}=(1-\sin \Psi) /(1+\sin \Psi)
$$

L'équation (11) peut être intégrée, et il apparait la constante $\mathrm{C} 1$ qui ne dépend pas du déplacement u et du rayon $r$ :

$$
\varepsilon_{r}^{p}=-\mathrm{n} \cdot \varepsilon_{\theta}^{p}+\mathrm{C} 1
$$

Nous supposons que la partie élastique de la déformation est négligeable dans cette zone, et nous utilisons les relations entre déformations et déplacements :

$$
\varepsilon_{\mathrm{r}}=\mathrm{d} \mathrm{w} / \mathrm{dr} \quad \varepsilon_{\mathrm{z}}=\mathrm{dv} / \mathrm{dz} \quad \varepsilon_{\theta}=\mathrm{w} / \mathrm{r}
$$

L'équation (13) peut être écrite en terme de déplacement par les relations (14), et nous pouvons intégrer entre les valeurs a et $r$ du rayon. Nous utilisons la notation $\mathrm{u}_{\mathrm{a}}$ pour le déplacement radial au niveau du forage, et la notation u pour le déplacement radial au rayon $r$. Nous trouvons :

$$
\begin{aligned}
& \operatorname{Ln}(r / a)=1 /(n+1) \cdot \operatorname{Ln}\left\{\left[u_{a} / a \cdot(1+n)\right.\right. \\
& -C 1] /[u / r \cdot(1+n)-C 1]\}
\end{aligned}
$$


A partir des relations (10) et (15), nous trouvons la relation liant la contrainte radiale, le déplacement au forage, et le déplacement u pour le rayon $r$ :

$$
\begin{aligned}
& 1 /(n+1) \cdot \operatorname{Ln}\left(\left[u_{a} / a \cdot(1+n)-C 1\right] /[u / r \cdot(1+n)\right. \\
& -C 1])=(1+n) /(1-N) \cdot \operatorname{Ln}\left(p / \sigma_{2}\right)
\end{aligned}
$$

A la limite de la première zone plastique, la contrainte radiale prend la valeur de la contrainte verticale (Fig. 3). L'expression (10) permet dans ces conditions de trouver l'extension de la première zone plastique:

$$
\mathrm{b}=\mathrm{a} \cdot[(-\gamma \mathrm{z}) / \mathrm{p}][1 /(\mathrm{N}-1)]
$$

\section{4}

\section{Seconde zone plastique}

Dans certaines conditions, une seconde zone plastique se développe entre la contrainte verticale et la contrainte circonférentielle, La condition d'équilibre vertical (7) donne la contrainte verticale à la profondeur $z$, sans force de surface :

$$
\sigma_{z}=-\gamma, z
$$

Dans le cas oủ la plasticité se développe dans cette zone, le critère de Mohr-Coulomb (5) donne un rapport $\mathrm{N}$ constant entre les contraintes circonférentielles et verticales. Ceci conduit à une valeur constante de la contrainte circonférentielle:

$$
\sigma_{\theta}=-\gamma \cdot 2 \cdot \mathrm{N}
$$

La condition d'équilibre (6) peut être écrite en fonction de $\mathrm{N}$ et peut être intégrée entre les valeurs c et $r$ du rayon. Nous utilisons la notation $\sigma_{r c}$ pour la contrainte radiale à la limite avec le domaine élastique, pour la valeur du rayon c. Nous avons alors la contrainte radiale pour un rayon $r$ quelconque telle que :

$$
\left(N \cdot \gamma \cdot z+\sigma_{\mathrm{rc}}\right) /\left(\mathrm{N}, \gamma \cdot z+\sigma_{\mathrm{r}}\right)=\mathrm{r} / \mathrm{C}
$$

La condition de plasticité non standard (2) donne le rapport entre les incréments de déformations plastiques circonférentielle et verticale. Nous utilisons les constantes d'intégration $\mathrm{C} 2$ et C3:

$$
\varepsilon_{z}^{p}=-n, \varepsilon_{\theta}^{P}+C 2 \quad \varepsilon_{r}^{p}=\mathrm{C} 3
$$

Nous négligeons la partie élastique de la déformation, et nous utilisons les relations (14) entre déformation et déplacement. La déformation radiale dans (21) peut être écrite en terme de déplacement et intégrée. Nous utilisons la constante d'intégration C4:

$$
\mathrm{u}=\mathrm{C} 3 \cdot \mathrm{r}+\mathrm{C} 4
$$

Cette équation, ainsi que la formule (20) donne la relation entre les contraintes et les déplacements dans la seconde zone plastique, avec $u_{c}$ qui est le déplacement pour le rayon c, limite externe de la seconde zone plas. tique et début de la zone élastique :

$$
\left(\mathrm{N} \cdot \gamma \cdot z+\sigma_{r c}\right) /\left(\mathrm{N} \cdot \gamma \cdot \mathrm{z}+\sigma_{\mathrm{r}}\right)=(\mathrm{u}-\mathrm{C} 4) /\left(\mathrm{u}_{\mathrm{c}}-\mathrm{C} 4\right)
$$

\section{5}

\section{Zone élastique}

L'analyse de la zone élastique est bien connue et peut être trouvée dans Cassan (1978) ou Baguelin et al. (1978). Nous développons cette analyse avec nos propres notations. Les contraintes radiales et circonférentielles s'écrivent alors:

$$
\begin{aligned}
\sigma_{r} & =(\lambda+2 \mu) \cdot \varepsilon_{r}+\lambda \cdot \varepsilon_{\theta}+\lambda \cdot \varepsilon_{z} \varepsilon_{\theta} \\
& =\lambda \cdot \varepsilon_{r}+(\lambda+2 \mu) \cdot \varepsilon_{\theta}+\lambda \cdot \varepsilon_{z}
\end{aligned}
$$

La condition d'équilibre horizontale (6) et les relations (14) donnent une équation différentielle du second ordre qui peut être intégrée avec la constante C5, le déplacement étant nul pour un rayon infini :

$$
\mathrm{u}=\mathrm{C} 5 / \mathrm{r}
$$

La relation (25) peut être introduite dans les équations élastiques (24). Pour un rayon infini, la contrainte radiale est liée à la contrainte verticale par. le coefficient des terres au repos $\mathrm{K}_{\alpha}$ si bien que les relations deviennent en terme de contrainte et de déformation :

$$
\begin{aligned}
& \sigma_{r}=-2 \mu \cdot C 5 / r^{2}-K_{0} \cdot \gamma \cdot z \\
& \sigma_{\theta}=2 \mu \cdot C 5 / r^{2}-K_{0} \cdot \gamma \cdot z \\
& \varepsilon_{r}=-C 5 / r^{2} \quad \varepsilon_{\theta}=C 5 / r^{2}
\end{aligned}
$$

\section{6}

\section{Équation de la courbe pressiométrique et condition de continuité entre les différentes zones}

Ces conditions dépendent de l'existence ou non de la seconde zone plastique. Nous allons d'abord examiner le cas plus général, où deux zones plastiques diffétentes se développent avec un écoulement plastique entre les axes verticaux et circonférentiels, puis le cas particulier où seule la première zone plastique existe.

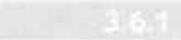 \\ Premier cas : deux zones plastiques se développent}

La limite entre les zones élastique et plastique est localisée à la valeur c du rayon. Nous connaissons le rapport $N$ entre les contraintes circonférentielles et verticales. La contrainte circonférentielle peut être écrite en fonction du rapport $\mathrm{N}$ par l'intermédiaire du critère de Mohr-Coulomb, et en fonction de C5 par les équations élastiques. En tenant compte des formules (26) nous trouvons la valeur de la constante $C 5$ et de $\sigma_{\text {rif }}$ qui est constant et indépendant du chargement p appliqué par le pressiomètre à la paroi du forage : $\sigma_{\text {tc }}$ est égal à la pression de fluage :

$$
\begin{gathered}
C 5=-c 2 \cdot\left(N-K_{0}\right) \cdot \gamma \cdot z /(2 \cdot \mu) \\
P_{f}=-\sigma_{r c}=\left(2 \cdot K_{0}-N\right) \cdot \gamma \cdot z
\end{gathered}
$$

Les relations (25), (27), (28), (29) peuvent être utlisées pour trouver le déplacement radial à la limite c :

$$
\mathrm{u}_{\mathrm{c}}=-\mathrm{c} \cdot\left(\mathrm{N}-\mathrm{K}_{0}\right) \cdot \gamma \cdot \mathrm{z} /(2 \cdot \mu)
$$



alors :

Pour la valeur c du rayon, les déformations sont

$$
\begin{aligned}
& \varepsilon_{\mathrm{rc}}=\left(\mathrm{N}-\mathrm{K}_{0}\right) \cdot \gamma, z /(2, \mu) \\
& \varepsilon_{\mathrm{\theta c}}=-\left(\mathrm{N}-\mathrm{K}_{0}\right) \cdot \gamma \cdot \mathrm{z} /(2, \mu)
\end{aligned}
$$

La constante C3 qui est égale à la déformation $\varepsilon_{\text {r }}$ dans la seconde zone plastique, est ainsi définie:

$$
\mathrm{C} 3=\left(\mathrm{N}-\mathrm{K}_{0}\right) \cdot \gamma \cdot z /(2, \mu)
$$

L'équation (20) peut s'appliquer entre les rayons plastiques b et $\mathrm{c}$. Avec la condition $\sigma_{\mathrm{rb}}$ égal à la contrainte verticale, $\sigma_{c e}$ égal à l'expression (29), et b donné par l'équation (17), on obtient finalement l'équation du second rayon plastique :

$$
\mathrm{c}=\mathrm{a} \cdot(\mathrm{N}-1) /\left[2\left(\mathrm{~N}-\mathrm{K}_{0}\right)\right] \cdot[(-\gamma \mathrm{z}) / \mathrm{p}]^{12 /(\mathrm{N}-1)]}
$$

A la limite entre la première et la seconde zone plastique, la valeur de la déformation plastique radiale peut être calculée par l'équation (15) et égalée à la constante C.3, ce qui donne :

$$
\begin{aligned}
& 1 /(1+n) \cdot\left\{C 1-n\left[(1+n) \cdot u_{a} / a-C 1\right]\right] \cdot(a / r)^{(1+n)} \\
& =\left(N-K_{0}\right) \cdot \gamma \cdot z /(2 \cdot \mu)
\end{aligned}
$$

Nous en déduisons la valeur de la constante C1:

$$
\begin{aligned}
& \mathrm{C} 1=\left[\mathrm{n} \cdot\left(\mathrm{u}_{\mathrm{a}} / \mathrm{a}\right) \cdot(1+\mathrm{n}) \cdot(-\gamma \cdot \mathrm{z} / \mathrm{p})^{\alpha}+(1+\mathrm{n})\right. \\
& \left.\left(\mathrm{N}-\mathrm{K}_{0}\right) \cdot \gamma \cdot \mathrm{z} /(2 \cdot \mu)\right] /\left[1+\mathrm{n}(-\gamma \cdot \mathrm{z} / \mathrm{p})^{\alpha}\right]
\end{aligned}
$$

avec $: a=(1+n) /(1-N)$

La relation (22) sur les déplacements dans la seconde zone plastique prise entre les valeurs b et $c$ du rayon donne le rapport entre les déplacements correspondants $u_{b}$ et $u_{c}$. La condition d'équilibre (23) de la seconde zone plastique donne le rapport entre les rayons b et c et nous trouvons :

$$
\mathrm{u}_{\mathrm{b}} / \mathrm{b}=\mathrm{u}_{\mathrm{c}} / \mathrm{b}+\mathrm{C} 3 \cdot\left(1-(\mathrm{N}-1) /\left[2\left(\mathrm{~N}-\mathrm{K}_{0}\right)\right]\right)
$$

Les conditions d'équilbre élastiques (30) donnent le déplacement $u_{c}$ au rayon $\mathrm{c}$, ce qui peut être introduit dans l'équation précédente et nous obtenons :

$$
\mathrm{u}_{\mathrm{b}} / \mathrm{b}=-\left(1-\mathrm{K}_{0}\right) \cdot \gamma \cdot \mathrm{z} /(2 \cdot \mu)
$$

La distribution des contraintes dans la première zone plastique (16) peut être utilisée entre les valeurs a et b du rayon, et nous trouvons la relation générale (38) qui est l'expression théorique de la courbe pressiométrique dans le cas où deux zones distinctes de plasticité se développent:

$$
\begin{aligned}
& \operatorname{Ln}\left[u_{a} / a \cdot(1+n)-C 1\right]=\alpha \cdot \operatorname{Ln}(-p)-\alpha, \operatorname{Ln}(\gamma \cdot z) \\
& +\operatorname{Ln}\left[\left(1-K_{0}\right) \cdot \gamma \cdot z \cdot(1+n) /(2 \cdot \mu)-C 1\right]
\end{aligned}
$$

Le terme $\mathrm{C} 1$ est très petit. Si nous le négligeons nous trouvons une relation linéaire entre les logarithmes du déplacement $u_{a}$ au niveau du forage, et la pression $p$ appliquée, dont la pente $\alpha$ est une fonction de l'angle de frottement interne $\Phi$ et de l'angle de frottement intergranulaire $\Phi_{u}$. La mesure de $\alpha$ et la connaissance de l'angle de frottement intergranulaire $\Phi_{u}$ permettent alors de déterminer l'angle de frottement interne $\Phi$.

En passant à l'exponentielle cette expression donne la relation contrainte déplacement pour l'essai pressiométrique :

$$
\begin{aligned}
& u_{a} / a=1 /(1+n) \cdot\left([-p /(\gamma \cdot z)]^{\alpha} \cdot\left(1-K_{0}\right) \cdot \gamma \cdot z .\right. \\
& (1+n) /(2 \mu)-C 1)+C 1
\end{aligned}
$$

\section{Second cas : la plasticité se développe seulement entre les directions circonférentielles et radiales}

Dans cette configuration, la seconde zone plastique disparaît, et le rayon b qui déterminait la limite entre les deux zones plastiques n'a plus de raison d'être. Il subsiste seulement dans ce cas le rayon a du forage, et le rayon c qui limite la zone plastique et la zone élastique.

Les contraintes au niveau du rayon c peut s'exprimer par les relations (26), ce qui donne

$$
\begin{gathered}
\sigma_{r c}=-2 \mu \cdot C 5 / c 2-K_{0} \cdot \gamma \cdot z \\
\sigma_{\theta c}=2 \mu \cdot C 5 / c 2-K_{0} \cdot \gamma \cdot z
\end{gathered}
$$

La condition de plasticité (8) appliquée à ces valeurs des contraintes donne la valeur de la déformation circonférentielle au rayon c qui est constante :

$$
C 5 / c 2=K_{0} \cdot \gamma \cdot z \cdot(1-N) /[(1+N) \cdot(2 \mu)]=\varepsilon_{\theta c}=-\varepsilon_{\gamma c}
$$

On en déduit la valeur des contraintes radiales et circonférentielles pour cette valeur du rayon qui sont constantes toutes deux; $\sigma_{r c}$ est égale à la pression de fluage :

$$
\begin{aligned}
& P_{f}=-\sigma_{r c}=2 \cdot K_{0} \cdot \gamma \cdot z /(1+N) \\
& \sigma_{\theta c}=-2 \cdot N \cdot K_{0} \cdot \gamma \cdot z /(1+N)
\end{aligned}
$$

La relation (10) appliquée pour le rayon c et la contrainte radiale $\sigma$ définie par la relation (42) donnent la valeur du rayon plastique c :

$$
\mathrm{c}=\mathrm{a} \cdot\left(-2 \cdot \mathrm{K}_{0} \cdot \gamma \cdot \mathrm{z} /[(1+\mathrm{N}) \cdot \mathrm{p}]\right]^{[1 /(\mathrm{N}-1)]}
$$

De la même façon que précédemment, on néglige les déformations élastiques devant les déformations plastiques, et la formule (13), dans laquelle on introduit la valeur des déformations (41), permet de trouver la nouvelle constante $\mathrm{C} 1$ de cet état d'équilibre :

$$
\mathrm{C} 1=\left[\mathrm{K}_{0} \cdot \gamma \cdot z \cdot(1-\mathrm{N}) \cdot(\mathrm{n}-1)\right] /[(1+\mathrm{N}) \cdot(2 \mu)]
$$

La formule (16) appliquée entre les rayons a et c donne la formule de la courbe pressiométrique :

$\operatorname{Ln}\left[\mathrm{u}_{\mathrm{a}} / \mathrm{a} \cdot(1+\mathrm{n})-\mathrm{C} 1\right]=\alpha \cdot \operatorname{Ln}(-\mathrm{p})-\alpha \cdot \operatorname{Ln}\left[\left(2-\mathrm{K}_{0} \cdot \gamma \cdot z\right)(1+\mathrm{N})\right.$ $+\operatorname{Ln}\left(K_{0} \cdot \gamma \cdot z \cdot(1-N) \cdot(1+n) /[2 \cdot \mu \cdot(1+N)]-C 1\right]$

Le terme C1 est très petit. Si nous le négligeons, nous trouvons une relation linéaire entre les logarithmes du déplacement $u_{s}$ au niveau du forage, et la pression p appliquée, dont la pente $\alpha$ est une fonction de l'angle de frottement interne $\Phi$ et de l'angle de frottement intergranulaire $\Phi_{\mu}$. La mesure de $\alpha$ et la connaissance de l'angle de frottement intergranulaire $\Phi_{\mu}$ permettent alors de déterminer l'angle de frottement interne $\Phi$.

En passant à l'exponentielle cette expression donne la relation contrainte de déplacement pour l'essai pressiométrique :

$\mathrm{u}_{\mathrm{a}} / \mathrm{a}=1 /(1+\mathrm{n}) \cdot\left[-\mathrm{p} \cdot(1+\mathrm{N}) /\left(2 \cdot \mathrm{K}_{0} \cdot \gamma \cdot \mathrm{z}\right)\right]^{\mathrm{a}}$ $\cdot\left(\mathrm{K}_{0} \cdot \gamma \cdot \mathrm{z} \cdot(1-\mathrm{N}) \cdot(1+\mathrm{n}) /[2 \cdot \mu \cdot(1+\mathrm{N})]-\mathrm{C} 1\right\}+\mathrm{C} 1$

La distinction entre les deux comportements possibles se fait sur la valeur de la contrainte radiale pour le rayon c. Pour le second cas de comportement, c'està-dire pour qu'une seule zone plastique existe seulement, il suffit que la contrainte radiale au rayon plastique 
c dépasse la valeur de la contrainte verticale en compression $\left(\sigma_{\mathrm{rc}}<\sigma_{z}\right)$ ou encore :

$$
-2 \cdot K_{0} \cdot \gamma \cdot z /(1+N)<-\gamma \cdot z
$$

ce qui simplifie en:

$$
K_{0}>=(1+N) / 2
$$

On retrouve alors la relation de Wood et al. (1977) pour avoir une seule zone plastique:

$$
\mathrm{K}_{0}>=1 /(1+\sin \Phi)
$$

\section{7}

\section{Pression de fluage et pression limite}

Deux cas peuvent se présenter selon qu'une ou deux zones plastiques se développent.

\subsection{1.}

\section{Premier cas : deux zones plastiques se développent}

Nous avons montré que la formule (29) donne la pression de fluage :

$$
P_{f}=\left(2 \cdot K_{0}-N\right) \cdot \gamma \cdot z
$$

Lorsque le volume injecté correspond au volume initial, u est égal à a/2, et la pression est égale à la pression limite conventionnelle. Si nous considérons maintenant l'équation (39), elle se transforme en :

$$
\operatorname{Plim}=\gamma \cdot z \cdot \sqrt[\alpha]{(1+n-2 \cdot C 1) \cdot \mu /\left[\left(1-K_{0}\right) \cdot(1+n) \cdot \gamma \cdot z-2 \cdot \mu \cdot C_{1}\right]}
$$

Dans cette relation, la pression limite est proportionnelle à la profondeur de l'essai, ce qui semble logique dans un sol frottant. En revanche, cette relation est sensiblement différente de celle de Amar et al. (1991):

$$
\mathrm{p}_{\mathrm{lim}}=250 \cdot 2^{\left(\Phi-24^{\vartheta} / 4\right.}+\mathrm{K}_{0} \cdot \gamma \cdot \mathrm{z}
$$

\section{Second cas : une zone plastique se développe}

Nous avons montré que la formule (42) donne la pression de fluage :

$$
\mathrm{P}_{\mathrm{f}}=2 \cdot \mathrm{K}_{0} \cdot \gamma \cdot \mathrm{z} /(1+\mathrm{N})
$$

Lorsque le volume injecté correspond au volume initial, $u_{\text {, }}$ est égal à $a / 2$, et la pression est égale à la pression limite conventionnelle. Si nous considérons maintenant l'équation (46), elle se transforme en :

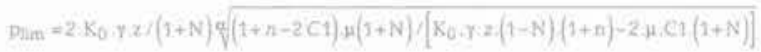

Dans cette relation, comme précédemment, la pression limite est proportionnelle à la profondeur de l'essai, ce qui semble logique dans un sol frottant.

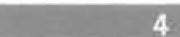

\section{Programme expérimental}

\section{1}

\section{Cas du sol avec deux zones plastiques}

Une série d'essais pressiométriques ont été réalisés sur le site de la construction du métro de Lyon, place Bellecour, en novembre 1986. Ce terrain est caractérisé par la présence de 0 à $6 \mathrm{~m}$ de remblais, de 7 à $12 \mathrm{~m}$ on trouve une grave beige propre correspondant aux allu-

\begin{tabular}{|c|c|c|c|c|c|c|c|c|}
\hline Essai & $\begin{array}{l}\text { Cote } \\
\text { (m) }\end{array}$ & $\begin{array}{l}\text { Module } \\
\text { d'Young } \\
\text { (kPa) }\end{array}$ & $\begin{array}{l}\text { Angle } \\
\text { frotte. } \\
\text { (degré) }\end{array}$ & $\begin{array}{c}\text { Pression } \\
\text { limite } \\
\text { exp. } \\
\text { (kPa) }\end{array}$ & $\begin{array}{l}\text { Pression } \\
\text { limite } \\
\text { CTRE4 } \\
\text { (kPa) }\end{array}$ & $\begin{array}{c}\text { Pression } \\
\text { limite } \\
\text { Théorie } \\
(\mathrm{kPa})\end{array}$ & $\begin{array}{c}\text { Ecart } \\
\text { Exp./ } \\
\text { CTRE4 } \\
(\%)\end{array}$ & $\begin{array}{c}\text { Ecart } \\
\text { Exp.l } \\
\text { Théorie } \\
(\%)\end{array}$ \\
\hline $\begin{array}{l}\text { WH_06 } \\
\text { WH_08 } \\
\text { WH_09 } \\
\text { WH_10 } \\
\text { WH_11 } \\
\text { WH_12 } \\
\text { WH_13 } \\
\text { WH_14 } \\
\text { WH_15 } \\
\text { WH_16 } \\
\text { WH_18 } \\
\text { WH_19 } \\
\text { WH_21 }\end{array}$ & $\begin{array}{r}6 \\
8 \\
9 \\
10 \\
11 \\
12 \\
13 \\
14 \\
15 \\
16 \\
18 \\
19 \\
21\end{array}$ & $\begin{array}{r}6000 \\
2000 \\
40000 \\
35050 \\
13660 \\
24560 \\
43630 \\
50800 \\
42240 \\
46060 \\
30080 \\
50000 \\
40000\end{array}$ & $\begin{array}{l}35 \\
30 \\
34 \\
45 \\
32 \\
31 \\
45 \\
45 \\
40 \\
35 \\
32 \\
33 \\
35\end{array}$ & $\begin{array}{r}520 \\
335 \\
1343 \\
2223 \\
765 \\
808 \\
2876 \\
3649 \\
1799 \\
1488 \\
1284 \\
2147 \\
1884\end{array}$ & $\begin{array}{r}1749 \\
786 \\
1499 \\
9604 \\
1096 \\
943 \\
9621 \\
9627 \\
4119 \\
1807 \\
1137 \\
1331 \\
1835\end{array}$ & $\begin{array}{r}545 \\
346 \\
1253 \\
2206 \\
823 \\
992 \\
2683 \\
2979 \\
2155 \\
1768 \\
1362 \\
1771 \\
11891\end{array}$ & $\begin{array}{r}236 \\
135 \\
12 \\
332 \\
43 \\
17 \\
234 \\
164 \\
129 \\
21 \\
-11 \\
-38 \\
-3 \\
106\end{array}$ & $\begin{array}{r}5 \\
3 \\
-7 \\
-8 \\
8 \\
23 \\
-7 \\
-18 \\
20 \\
19 \\
6 \\
-18 \\
0\end{array}$ \\
\hline
\end{tabular}
vions du Rhône, et de 13 à $22 \mathrm{~m}$ une grave rougeâtre propre qui correspond aux alluvions de la Saône. Les résultats des essais nous ont été aimablement fournis par le CETU de Bron et sont indiqués dans le tableau I.

TABLEAU1 Comparaison des pressions limites expérimentales et théoriques pour la grave de Lyon.

Comparison between experimental and theoretical limit pressures for the gravel of Lyon. 
Cette série d'essais a été réalisée avec un tube lanterné, battu dans le sol. La pente $\alpha$ de la droite reliant le logarithme de la déformée du forage, au logarithme de la pression (Fig. 5), pour les points au-delà du fluage, permet de donner une première approche de l'angle de frottement interne à partir de la relation (38). La superposition de la courbe théorique (39) et de la courbe expérimentale (Fig. 6) permet d'affiner l'angle de frottement interne du sol qui est indiqué dans la quatrième colonne du tableau 1. Le module d'élasticité a été déterminé à l'aide de la pente du cycle de déchargement rechargement que l'on voit sur la figure 6. Les essais triaxiaux qui ont été réalisés à l'IRIGM à Grenoble ont indiqués un angle de frottement moyen de $41,3^{\circ}$, avec $19 \mathrm{kPa}$ de cohésion, avec un angle de frottement intergranulaire de $30^{\circ}$. Le module d'Young moven est de $28000 \mathrm{kPa}$, et le coefficient de Poisson de 0,364 , ce qui donne un coefficient $\mathrm{K}_{0}$ de 0,572. L'échantillon était remanié, reconsolidè et drainé à la masse volumique initiale moyenne de $1,8 \mathrm{~g} / \mathrm{cm}^{3}$. La pression limite théorique est donnée par la formule (51), et la pression limite CTRE4 (Comité Technique Régional Européen n 4, Amar et al. 1991) est donnée par la relation (52). On constate systématiquement un meilleur accord entre la théorie que nous proposons et l'expérience, qu'entre la formule usuelle du CTRE4 et l'expérience, au point que l'écart moyen se ramène dans notre cas à $12 \%$, alors qu'il est de $106 \%$ dans le cas de la relation du CTRE4.

\section{2}

\section{Cas du sol avec une zone plastique}

Une série d'essais pressiométriques ont été réalisés sur le site de la construction du collecteur de CauderanNaujac à Bordeaux, en janvier 1987. Ce site est caractérisé par une couche d'argiles de 0 à $7 \mathrm{~m}$, puis une couche de sable bien gradulé à partir de $8 \mathrm{~m}$ de profondeur. Les résultats des essais nous ont été aimablement fournis par le CETU de Bron, et sont indiqués sur le tableau II.

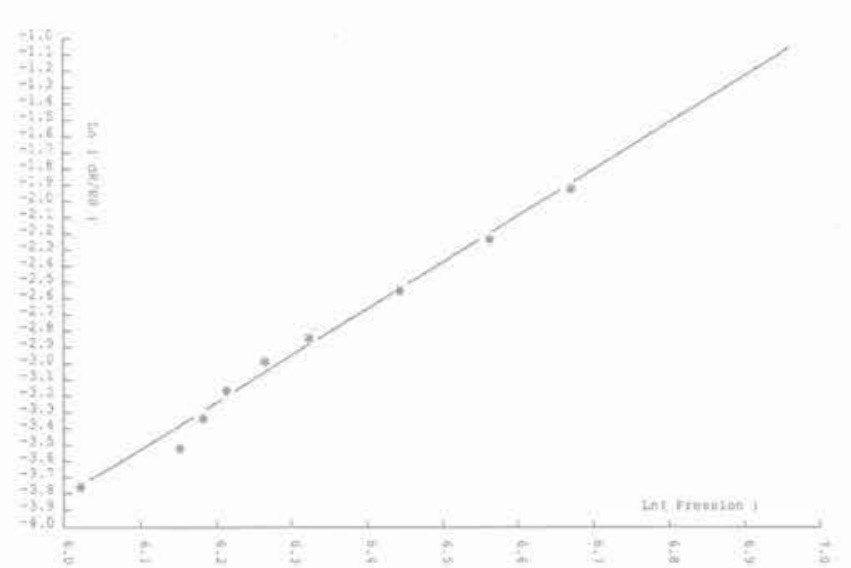

AG.5 Transformation linéaire de la courbe pressiométrique pour l'essai WH_09 à $9 \mathrm{~m}$ de profondeur, dans la grave de Lyon. Linear transformation of the pressurementer curve for the WH_09 test with $9 \mathrm{~m}$ depth in the gravel of Lyon:

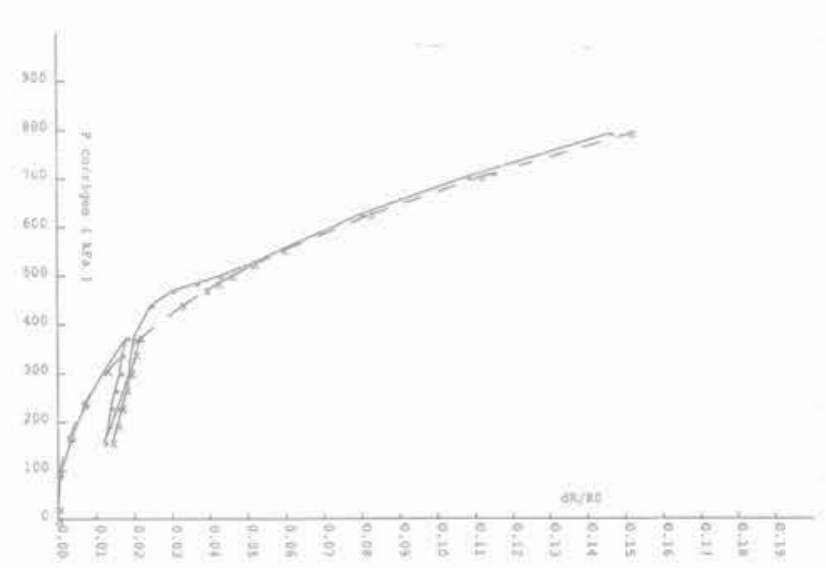

FG.6 Comparaison des courbes expérimentales et théoriques pour l'essai WH_09 à $9 \mathrm{~m}$ de profondeur, dans la grave de Lyon.

Comparison between experimental and theoretical curves for the WH_O9 test with $9 \mathrm{~m}$ depth in the gravel of Lyon.

TABLEAUII Comparaison des pressions limites expérimentales et théoriques pour le sable de Bordeaux.

Comparison between experimental and theoretical curves for the PR2_11 test with 11 on depth in the sand of Bordeaux.

\begin{tabular}{|c|c|c|c|c|c|c|c|c|}
\hline Essai & $\begin{array}{l}\text { Cote } \\
\text { (m) }\end{array}$ & $\begin{array}{l}\text { Module } \\
\text { d'Young } \\
(\mathrm{kPa})\end{array}$ & $\begin{array}{l}\text { Angle } \\
\text { frotte. } \\
\text { (degré) }\end{array}$ & $\begin{array}{c}\text { Pression } \\
\text { limite } \\
\text { exp. } \\
\text { (kPa) }\end{array}$ & $\begin{array}{c}\text { Pression } \\
\text { limite } \\
\text { CTRE4 } \\
\text { (kPa) }\end{array}$ & $\begin{array}{c}\text { Pression } \\
\text { limite } \\
\text { Théorie } \\
(\mathrm{kPa})\end{array}$ & $\begin{array}{c}\text { Ecart } \\
\text { Exp.l } \\
\text { CTRE4 } \\
(\%)\end{array}$ & $\begin{array}{c}\text { Ecart } \\
\text { Exp.l } \\
\text { Théorie } \\
(\%)\end{array}$ \\
\hline $\begin{array}{l}\text { PR1_8 } \\
\text { PR1_9 } \\
\text { PR1_11 } \\
\text { PR1_12 } \\
\text { PR1_13 } \\
\text { PR1_14 } \\
\text { PR2_8 } \\
\text { PR2_9 } \\
\text { PR2_10 } \\
\text { PR2_11 } \\
\text { PR2_12 } \\
\text { PR2_13 } \\
\text { PR2_14 } \\
\text { Moy. }\end{array}$ & $\begin{array}{r}8 \\
9 \\
11 \\
12 \\
13 \\
14 \\
8 \\
9 \\
10 \\
11 \\
12 \\
13 \\
14\end{array}$ & $\begin{array}{l}20300 \\
17060 \\
55800 \\
33880 \\
12780 \\
29200 \\
59000 \\
25700 \\
40600 \\
29000 \\
36670 \\
14500 \\
25000\end{array}$ & $\begin{array}{l}40 \\
37 \\
29 \\
27 \\
33 \\
34 \\
31 \\
31 \\
35 \\
30 \\
28 \\
26 \\
24\end{array}$ & $\begin{array}{rr}1 & 440 \\
1 & 180 \\
1 & 430 \\
1 & 020 \\
970 \\
1380 \\
1 & 250 \\
1 & 040 \\
1 & 540 \\
1 & 140 \\
1 & 240 \\
& 630 \\
& 720\end{array}$ & $\begin{array}{r}4090 \\
2480 \\
710 \\
540 \\
1320 \\
1550 \\
930 \\
940 \\
1790 \\
1120 \\
620 \\
490 \\
390\end{array}$ & $\begin{array}{r}1400 \\
1160 \\
1260 \\
980 \\
990 \\
1510 \\
1280 \\
990 \\
1580 \\
1080 \\
1070 \\
740 \\
810\end{array}$ & $\begin{array}{r}184 \\
110 \\
-50 \\
-47 \\
36 \\
12 \\
-26 \\
-10 \\
16 \\
-2 \\
-50 \\
-22 \\
-46 \\
47\end{array}$ & $\begin{array}{r}-3 \\
-2 \\
-12 \\
-4 \\
2 \\
9 \\
2 \\
-5 \\
3 \\
-5 \\
-14 \\
17 \\
12 \\
7\end{array}$ \\
\hline
\end{tabular}


Cette série d'essai a été réalisée avec un tube lanterné, battu dans le sol. La pente $\alpha$ de la droite reliant le logarithme de la déformée du forage, au logarithme de la pression (Fig. 7), pour les points au-delà du fluage, permet de donner une première approche de l'angle de frottement interne à partir de la relation (45). La superposition de la courbe théorique (46), et de la courbe expérimentale (Fig. 8) permet d'affiner l'angle de frottement interne du sol qui est indiqué dans la quatrième colonne du tableau II. On remarque nettement sur la figure 8 le décalage initial entre la courbe expérimentale et la courbe théorique, qui sont mises en coincidence sur le cycle. Cet écart est dû au remaniement initial du sol par le procédé de fonçage. Le module d'élasticité a été déterminé à l'aide de la pente du cycle de déchargement-rechargement que l'on voit sur la figure 8. Les essais triaxiaux, qui ont été réalisés à l'IRIGM à Grenoble, ont indiqué un angle de frottement moyen de $35.9^{\circ}$, sans cohésion, avec un angle de frottement intergranulaire de $26^{\circ}$. Le module d'Young moyen est de $30000 \mathrm{kPa}$ et le coefficient de Poisson de 0,436 , ce qui donne un coefficient $K_{0}$ de 0,773 . L'échantillon était remanié, reconsolidé et drainé à la masse volumique initiale moyenne de $1,8 \mathrm{~g} / \mathrm{cm}^{3}$. La pression limite théorique est donnée par la formule (51), et la pression limite CTRE4 (Comité Technique Régional Européen n4. Amar et al. 1991) est donnée par la relation (54).

On constate ici un meilleur accord entre la théorie que nous proposons et l'expérience, qu'entre la formule usuelle du CTRE4 et l'expérience, au point que l'écart moyen se ramène dans notre cas à $7 \%$, alors qu'il est de $47 \%$ dans le cas de la relation du CTRE 4.

\section{5}

\section{Calcul numérique de l'équilibre autour du pressiomètre}

Nous utilisons le programme GAIAEF qui utilise des éléments quadrilatères à 16 nœuds. Nous avons intro- duit la loi de comportement MCK élasto-plastique (Monnet, 1992) qui tient compte de l'élasticité et de la plasticité avec dilatance non standard, ainsi que du chargement réellement tridimensionnel. Le maillage éléments finis comprend 48 éléments quadrilatères à 16 nceuds. Il y a 5 nœuds sur chaque coté de ces éléments. Les paramètres utilisés par le modèle sont indiqués dans le tableau III ci-après.

TABLEAUIII Les paramètres utilisés dans le calcul par éléments finis.

The soil parameters used in the finite element calculation.

\begin{tabular}{|c|c|c|}
\hline Paramètres & $\begin{array}{l}\text { Bellecour } \\
\text { WH_09 }\end{array}$ & $\begin{array}{l}\text { Bordeaux } \\
\text { PR2_11 }\end{array}$ \\
\hline $\begin{array}{l}\text { Indice des vides } e_{0} \\
\text { Coefficient de Poisson v } \\
\text { Indice de gonflement } C_{s} \\
\text { Indice de compression } C_{c} \\
\text { Cohésion } C \\
\text { Angle de frottement } \Phi \\
\text { Angle de frottement } \\
\text { intergranulaire } \Phi_{\mu} \\
\text { Rapport de rupture } R_{i}\end{array}$ & $\begin{array}{l}0,645 \\
0,364 \\
0,008 \\
0,013 \\
0 \\
33^{\circ} \\
\\
30^{\circ} \\
0,8\end{array}$ & $\begin{array}{l}0,550 \\
0,420 \\
0,007 \\
0,009 \\
0 \\
30^{\circ} \\
\\
26^{\circ} \\
0,75\end{array}$ \\
\hline
\end{tabular}

La correspondance entre la courbe analytique donnée par la relation (39) et le calcul par éléments finis est indiquée sur la figure 9, dans le cas de l'essai WH_09 de Bellecour. La correspondance entre la courbe analytique donnée par la relation (46) et le calcul par éléments finis est indiquée sur la figure 10, dans le cas de I'essai PR2 11 de Bordeaux. On constate une bonne coïncidence entre les courbes analytiques, calculées par éléments finis jusqu'à 7 à $8 \%$ de déformation. L'écart ultérieur peut être considéré comme un défaut du calcul numérique, qui manque de rééquilibrage quand des zones plastiques importantes se développent. Cependant, l'écart en déformation entre l'analyse et le calcul reste limité à $2 \%$ au maximum, et permet de valider les formules théoriques présentées.

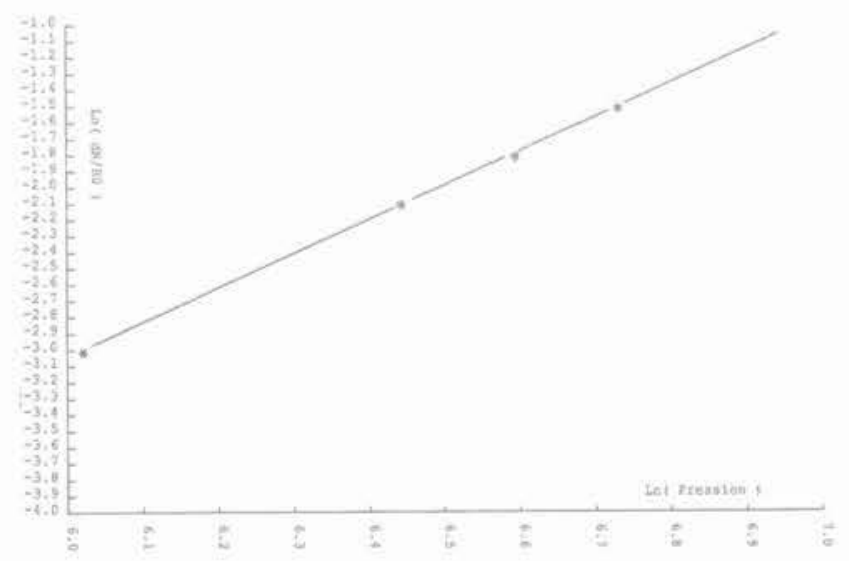

F10.7 Transformation linéaire de la courbe pressiométrique pour l'essai PR2 11 à $11 \mathrm{~m}$ de profondeur dans le sable de Bordeaux.

Linear transformation of the pressuremeter curve for the PR2 11 test with $11 \mathrm{~m}$ depth in the sand of Bordeaux.

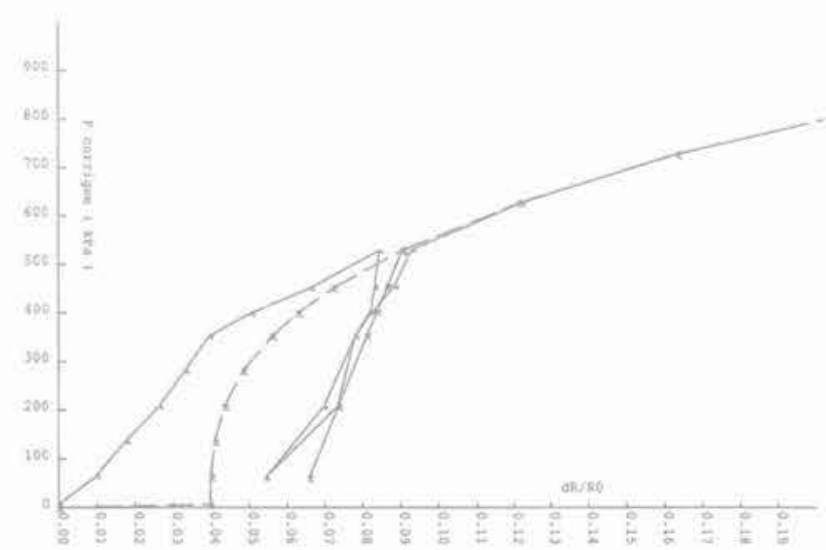

FG. 8 Comparaison des courbes expérimentales et théoriques pour l'essai PR2 11 à $11 \mathrm{~m}$ de profondeur dans le sable de Bordeaux. Comparison between experimental and theoretical curves for the PR2 11 test with $11 \mathrm{~m}$ depth in the sand of Bordeaux. 


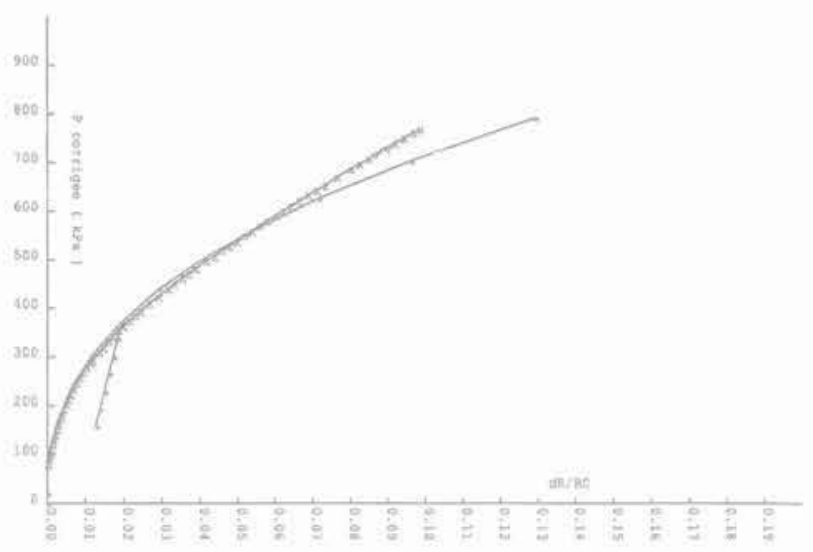

FIG.9 Comparaison des courbes analytiques et calculées pour l'essai WH_09 à $9 \mathrm{~m}$ de profondeur dans la grave de Lyon. Comparison between analytical and numerical curves for the WH_09 test with $9 \mathrm{~m}$ depth in the gravel of Lyon.

On remarque également que l'étendue de la zone linéaire et élastique du début de l'essai est très peu étendue. Elle est limitée théoriquement à $128 \mathrm{kPa}$ pour WH_09, alors que l'expérience donne une valeur de $350 \mathrm{kPa}$, elle est limitée théoriquement à $174 \mathrm{kPa}$ pour PR2_11, alors que l'expérience donne une valeur de $400 \mathrm{kPa}$. L'étendue très limitée de cette zone élastique est confirmée par le calcul éléments finis (Fig. 9 et 10). En revanche, le module élastique du sol est confirmé par la superposition des cycles expérimentaux et analytiques (Fig. 6 et 8).

\section{6}

\section{Conclusion}

Nous avons présenté une théorie nouvelle concernant l'essai pressiométrique. Celle-ci montre que la zone élastique initiale du chargement pressiométrique est

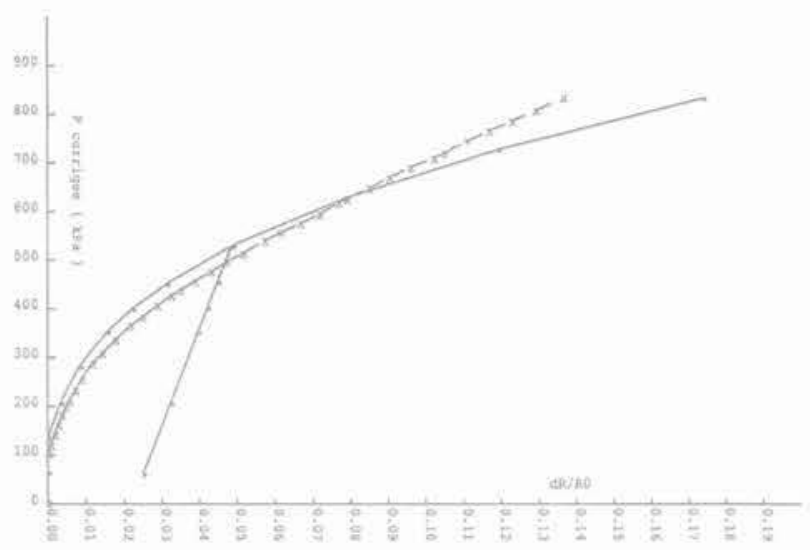

FIG. 10 Comparaison des courbes analytiques et calculées pour l'essai PR2_11 à $11 \mathrm{~m}$ de profondeur dans le sable de Bordeaux. Comparison between analytical and numerical curves for the PR2 11 test with $11 \mathrm{~m}$ depth in the sand of Bordeaux.

très limitée dans le cas du sol pulvérulent. Le module pressiométrique que l'on utilise habituellement est donc entaché d'une erreur due à la présence de déformations plastiques. Ces déformations sont mises en évidence en faisant un cycle de déchargement-rechargement qui montre alors une déformation permanente du sol. Cependant le module d'élasticité du sol peut être valablement mesuré par la pente du cycle de déchargementrechargement.

Le recouvrement des courbes analytique et expérimentale permet de déterminer précisément l'angle de frottement interne du sol, dans la mesure où on connaît l'angle de frottement intergranulaire, les formules théoriques ayant par ailleurs été validées par le calcul numérique.

\section{$\overline{\text { Bibliographie }}$}

Amar S., Clarke B.G.F, Gambin M.P., Orr T.L.L. Utilisation des essais pressiométriques pour le dimensionnement des fondations en Europe, Rapport sur l'état des connaissances établi par le Comité Technique Européen de la SIMSTF. Première partie: pressiométre Ménard et pressiometre autoforeur. Comité Technique Régional Européen n०4, Pressiomètres, A.A. Balkema, pp, 25-48, 1991

Baguelin F., Jezequel J.F., Shields D.H. - The pressuremeter and foundation engineering, Trans Tech Publication, 1978.

Cassan M. - Les essais in situ en mécanique des sols, Vol. 1, Paris, Eyrolles, 1978

Chaffois S.. Monnet J. - Modèle de comportement du sable au cisaillement dans un état tridimensionnel de contrainte et de déformation. Revue Française de Géotechnique, $\mathrm{n}^{\circ} 32$, pp. $59-69,1985$

Chaffois S., Monnet J. - Model of sand behaviour towards shearing and compressibility in 3 dimensional condition of stress and strain, Constitutive Equations for Granular and Non Cohesive Soil, Cleveland, Balkema, pp. 169181, 1989.
Gaiatech - Procédé d'essai de forage, Brevet Francals d'lnvention. $n^{\circ} 8909674$, déposé à Lyon le 12.07.1989.

Gambin M. - Calcul d'une fondation profonde en fonction des résultats pressiometrique, Sols Solls, $n^{\circ} 7,1963$.

Gambin M. - Vingt ans d'usage du pressiomètre en Europe, Congès europeen de Mécanique des Sols et des Travaux de Fondation, Brighton. 1979 .

Gambin M. - Calculation of foundation subjected to horizontal forces using pressuremeter data, Sols Soils, $n^{\circ}$ 30/31, 1979

Hughes F, Wroth C.P. Windle D. - Pressuremeter test in sands, Geotechnique, $\mathrm{n}^{\circ} 4$, pp. 455 477, 1977 .

Menard L. - Pressiomètre, Brevet Francais d'Invention. $n^{\circ} 1.117 .983$, déposé le 19 janvier 1955.

Menard L. - Dispositif d'étude de la déformation sous charge d'un milieu homogène, Brevet Français d'Invention, $\mathrm{N}^{\circ} 1.234 .756$, déposé le 15 mai 1959.

Monnet J - Theoretical study of elasto plastic equilibrium around pressuremeter in sands,
3 th International Symposium on pressureme. ters, Oxford, pp. 137-148. 1990.

Monnet J. - Equations constitutives du sol utilisant des paramétres géotechniques, Collogue René Houper, Nancy, pp. 171-180, 1992.

Monnet J. Chaffois S., Chapeau C.. Mohkam. Theoritical and experimental studies of a tunnel face in a gravel site. Part I: Basic theoritica approaches, Numerical Model in Geomechanics, NUMOG III, Niagara Falls, 1989.

Monnet J, Chaffois S., Chapeau C., Mohkam.Theoritical and experimental studies of a tunnel face in a gravel site. Part Il: Experimental and numerical analysis, Numerical Model in Geomechanics, NUMOG III. Niagara Falls, 1989,

Monnet J., Khlif J. - Theoretical and experimental study of equilibrium around pressuremeter. 4th International Symposium on Numerica. Models in Geomechanics, Swansea, 1992

Rowe. - The stress dilatancy relation for static equilibrium, Proc: Roy. Soc. 269. pp. 500-527, 1962.

Wood D.M. Wroth P.C. - Some laboratory experiments related to the results of pressuremeter tests, Geotechnique, 27, n² 2, pp. 181-201, 1977. 